\title{
Diagnosing plasma magnetization in inertial confinement fusion implosions using secondary deuterium-tritium reactions
}

\author{
H. Sio, ${ }^{1, a)}$ J. D. Moody, ${ }^{1}$ D. D. Ho, ${ }^{1}$ B. B. Pollock, ${ }^{1}$ C. A. Walsh, ${ }^{1}$ B. Lahmann, ${ }^{2}$ D. J. Strozzi, ${ }^{1}$ G. E. Kemp, ${ }^{1}$
} W. W. Hsing, ${ }^{1}$ A. Crilly, ${ }^{3}$ J. P. Chittenden, ${ }^{3}$ and B. Appelbe ${ }^{3}$

1) Lawrence Livermore National Laboratory, Livermore, CA, 94550 USA

${ }^{2)}$ Plasma Science 83 Fusion Center, Massachusetts Institute of Technology, Cambridge, Massachusetts 02139 USA

3) The Centre for Inertial Fusion Studies, The Blackett Laboratory, Imperial College, London SW7 $2 A Z$, United Kingdom

(Dated: 26 March 2021)

\begin{abstract}
Diagnosing plasma magnetization in inertial-confinement-fusion implosions is important for understanding how magnetic fields affect implosion dynamics, and to assess plasma conditions in magnetized implosion experiments. Secondary deuterium-tritium (DT) reactions provide two diagnostic signatures to infer neutronaveraged magnetization. Magnetically confining fusion tritons from deuterium-deuterium (DD) reactions in the hot spot increases their path lengths and energy loss, leading to an increase in the secondary DT reaction yield. In addition, the distribution of magnetically-confined DD-triton is anisotropic, and this drives anisotropy in the secondary DT-neutron spectra along different lines of sight. Implosion parameter space as well as sensitivity to applied B-field, fuel $\rho$ R, temperature, and hot-spot shape will be examined using Monte-Carlo and 2-D radiation-magnetohydrodynamic simulations.
\end{abstract}

\section{INTRODUCTION}

An externally-applied magnetic field can reduce thermal conduction and magnetically confine fast ions in a plasma. Both effects ease the physics requirements needed to reach ignition in a high-performing inertial confinement fusion (ICF) implosion ${ }^{1}$ by reducing heat loss and increasing DT- $\alpha$ energy deposition in the hot $\operatorname{spot}^{2,3}$. The development of a magnetized ICF platform ${ }^{4}$ is underway at the National Ignition Facility (NIF), requiring new diagnostics and scientific understanding of how Bfields affect implosion dynamics. Sensitive measurements are needed to validate MHD models, and to demonstrate key physics such as fast-ion confinement and magnetic flux compression in a magnetized hot spot.

The presence of compressed magnetic fields in an inertially-confined plasma can be indirectly inferred from increases in temperature and nuclear yields ${ }^{5}$, although this does not provide a quantitative magnetization measurement. A more direct probe of B-field compression can be made using proton deflectometry in spherical ${ }^{6}$ and cylindrical $^{7}$ geometries, but requires an additional target and diverting laser beams from the primary target.

One self-emission diagnostic approach to measure plasma magnetization makes use of secondary deuteriumtritium (DT) reactions ${ }^{8}$ in an implosion with deuterium fuel. Applied magnetic field can confine fast ions in the plasma, increasing these particles' energy loss and fusion probability. Both the secondary yield ratio and secondary neutron spectra can be used to diagnose neutronaveraged magnetization. This technique has been applied to cylindrical plasmas ${ }^{9,10}$, but has not been discussed in depth for spherical implosions.
This work describes the applications of secondary DT reaction in diagnosing plasma magnetization in ICF implosions, and the ICF parameter space for which this technique can be applied. Sec.II introduces secondary reactions and the Monte-Carlo model used in this work. Sec.III and Sec.IV discuss the application of secondary yield ratio and secondary neutron spectra, respectively. Sec.V outlines the parameter space for which this technique can be applied, and compares the predictions between Monte-Carlo calculations and 2-D radiationmagnetohydrodynamic (MHD) simulations.

\section{SECONDARY REACTIONS}

In a deuterium plasma, the two branches of the deuterium-deuterium (DD) reactions occur with approximately equal probability ${ }^{11}$. The first branch of the DD reaction produces a $2.45-\mathrm{MeV}$ neutron (DD-n), which we will refer to as a primary neutron.

$$
\mathrm{D}+\mathrm{D} \rightarrow \mathrm{n}(2.45 \mathrm{MeV})+{ }^{3} \mathrm{He}(0.82 \mathrm{MeV})
$$

The second branch of the DD reaction produces a 1.01$\mathrm{MeV}$ triton (DD-t) ${ }^{11}$.

$$
\mathrm{D}+\mathrm{D} \rightarrow T(1.01 \mathrm{MeV})+\mathrm{p}(3.02 \mathrm{MeV})
$$

As this DD-t leaves the fuel, it has a probability to undergo a DT fusion reaction with the background deuterons, producing a neutron and an alpha particle ${ }^{8}$. We refer to the neutron from this second fusion reaction as a secondary neutron (DT-n).

$$
\begin{array}{r}
\mathrm{D}+\mathrm{T}(\leq 1.01 \mathrm{MeV}) \rightarrow \mathrm{n}(11.9-17.2 \mathrm{MeV}) \\
+\alpha(6.7-1.4 \mathrm{MeV})
\end{array}
$$


Because the reactant DD-t can have a range of initial energies, the secondary DT-n produced also has a wide energy range. Typically, the ratio of secondary DT-n to primary DD-n $Y_{D T} / Y_{D D}$ is between $10^{-4}-10^{-2}$, depending on fuel $\rho \mathrm{R}$ and plasma temperature ${ }^{8}$. Areal density $(\rho \mathrm{R})$, the product of plasma density $\rho$ and radius $R$, is an important measure of compression in ICF implosions.

\section{A. Monte-Carlo model}

Secondary yield ratios and spectra are calculated in this work using a custom Monte-Carlo model to simulate DD-t trajectories within the fuel. Within the defined fuel volume, profiles of the magnetic field, one electron species, and at least one ion species are defined. The simulations discussed in later sections assumed static and uniform profiles for temperature, density, and B-field, and the B-field is axial along the z-direction. This model does not include ion scattering, as secondary yield is primarily a function of DD-t energy loss (path-length integral) in the fuel. The validity of these assumptions as compared to 2-D simulations of realistic ICF implosions with MHD models is discussed in Sec.V. Ion energy loss in the fuel is calculated using the Li-Petrasso model ${ }^{12}$.

In the main Monte-Carlo simulation, DD-t are generated within the fuel volume. At each time step, DD-t are transported through the fuel volume, accounting for energy loss and B-field. Adaptive time steps are used to better resolve the DD-t' gyroradii as well as spatial regions with high gradients. The trajectory calculation is stopped when the DD-t leaves the fuel volume, has no remaining energy, or exceeds a user-specified time (e.g. plasma confinement time).

In the post-processing stage, secondary yield ratio and neutron spectra are calculated from these simulated DD$\mathrm{t}$ trajectories. The probability that a DD-t undergoes a secondary DT reaction at time $t_{0}$ over a time interval $\Delta t$ is given by:

$$
P_{t=t_{0}}=n_{D} \sigma_{D T} v_{t} \Delta t\left(1-P_{t<t_{0}}\right)
$$

where $n_{D}$ is the local background deuteron number density, $\sigma_{D T}$ is the DT reaction cross section, and $v_{t}$ is the DD-t velocity. This DD- $t$ can only undergo a fusion reaction at this time step if it has not already fused earlier in its trajectory, and this is represented by the factor $1-P_{t<t_{0}}$. Summing the probabilities over all time steps gives the secondary yield ratio for one trajectory. Averaging over many DD-t trajectories gives the secondary yield ratio sampled over the entire fuel volume. In a typical simulation, $10^{5}$ DD-t particle trajectories (corresponding to $\sim 10^{7}$ secondary DT-n) are simulated, which is sufficient for convergence (with statistical uncertainty $\sim 1 \%$ ). Results from this model have been validated against analytic and numerical calculations ${ }^{8,13}$.

A similar approach is used to calculate the secondary neutron spectra. At different times, a back-

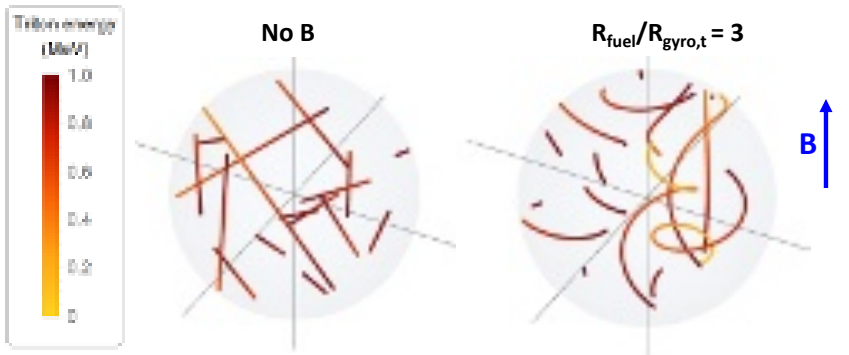

FIG. 1: Example simulated DD-t trajectories in a uniform plasma with a radius of $250 \mu \mathrm{m}$, and a deuteron fuel $\rho \mathrm{R}$ of $58 \mathrm{mg} / \mathrm{cm}^{2}$. The color scale indicates the energies of the DD-t as they are transported through the plasmas. On the left, no external B-field is applied.

On the right, a $2.14 \mathrm{kT}$ B-field is applied.

ground deuteron with a random velocity vector is sampled from a Maxwellian population based on local plasma temperature ${ }^{14}$, and a secondary DT-n is generated. For the two-body DT reaction, the energy of the DT-n is kinematically constrained by the reactants' velocities and the DT-n's direction. This information along with the DT differential cross section are used to calculate DT-n spectra along specific lines of sight.

Figure 1 shows example DD-t trajectories from a typical Monte-Carlo simulation. When no magnetic field is applied, DD-t in a moderate- $\rho \mathrm{R}$ fuel leave the plasma, losing some energy along the way. If the fuel $\rho \mathrm{R}$ is sufficiently high that the DD-t are completely stopped in the fuel, the DD-t are collisionally confined. If a sufficiently strong magnetic field is applied such that $R_{\text {gyro, },}$, the gyroradius of a $1.01 \mathrm{MeV}$ DD-t, is comparable to $R_{f u e l}$, the plasma radius, a small fraction of the DD-t population with large velocity component perpendicular to the applied B-field can be magnetically confined. These DD-t spend more time in the plasma and lose more energy in the plasma, significantly increasing their probability to undergo a secondary reaction.

$R_{\text {fuel }} / R_{\text {gyro,t }}$ is used in this work because it is a physically-intuitive, dimensionless parameter. In the context of describing an ICF implosion with initial radius $R_{0}$, final radius $R$, initial magnetic field $B_{0}$, final magnetic field $B$, and convergence ratio $C R=R / R_{0}$ :

$$
\begin{aligned}
R_{\text {fuel }} / R_{\text {gyro }, t} & =5.4 \times 10^{-6} B R[G \cdot \mathrm{cm}] \\
& =5.4 \times 10^{-6} R_{0}[\mu \mathrm{m}] B_{0}[T] C R
\end{aligned}
$$

$R_{\text {fuel }} / R_{\text {gyro,t }}$ is linearly proportional to the magnetic confinement parameter $B R$ (the product of magnetic field $B$ and radius $R$ ). The first equality makes use of the relationship $R_{g y r o, t}=m_{t} v_{t} / e B$, where $m_{t}$ and $v_{t}$ are the mass and velocity of an $1.01 \mathrm{MeV}$ triton. The second equality assumes that the magnetic field is frozen into the plasma such that the compressed magnetic field scales as $B=B_{0}(C R)^{2}$. 


\section{SECONDARY YIELD RATIO}

This section discusses the sensitivity of the secondary yield ratio $Y_{D T} / Y_{D D}$ as a function of fuel $\rho \mathrm{R}$ and Bfield. Figure 2 shows the simulated $Y_{D T} / Y_{D D}$ for a uniform, spherical plasma with a radius of $250 \mu \mathrm{m}$ and temperature of $5 \mathrm{keV}$, for different applied B-field. At $R_{\text {fuel }} / R_{\text {gyro,t }}=1(\mathrm{~B}=0.72 \mathrm{kT})$, there is little enhancement in $Y_{D T} / Y_{D D}$ as compared to the no-B baseline because fusion products are weighted toward the outer radius due to the volume effect. $Y_{D T} / Y_{D D}$ enhancement becomes significant when $R_{\text {fuel }} / R_{\text {gyro, } t} \geq 2$.

Figure 2 also illustrates three distinct regimes based on fuel $\rho$ R. Below $5 \mathrm{mg} / \mathrm{cm}^{2}$, the DD-t path length in the fuel volume is increased due to gyromagnetic effect, but there is no significantly increased DD-t energy loss, so there is little change to the DT cross section. Between $5-100 \mathrm{mg} / \mathrm{cm}^{2}$, the increase in DD-t path length in the fuel in combination with the moderate fuel $\rho \mathrm{R}$ is sufficient to significantly lower DD-t energies (see Fig.1), leading to an additional enhancement due to an increased DT cross section. Above $100 \mathrm{mg} / \mathrm{cm}^{2}$, a significant fraction of DD$\mathrm{t}$ are already collisionally confined by the fuel $\rho \mathrm{R}$, and
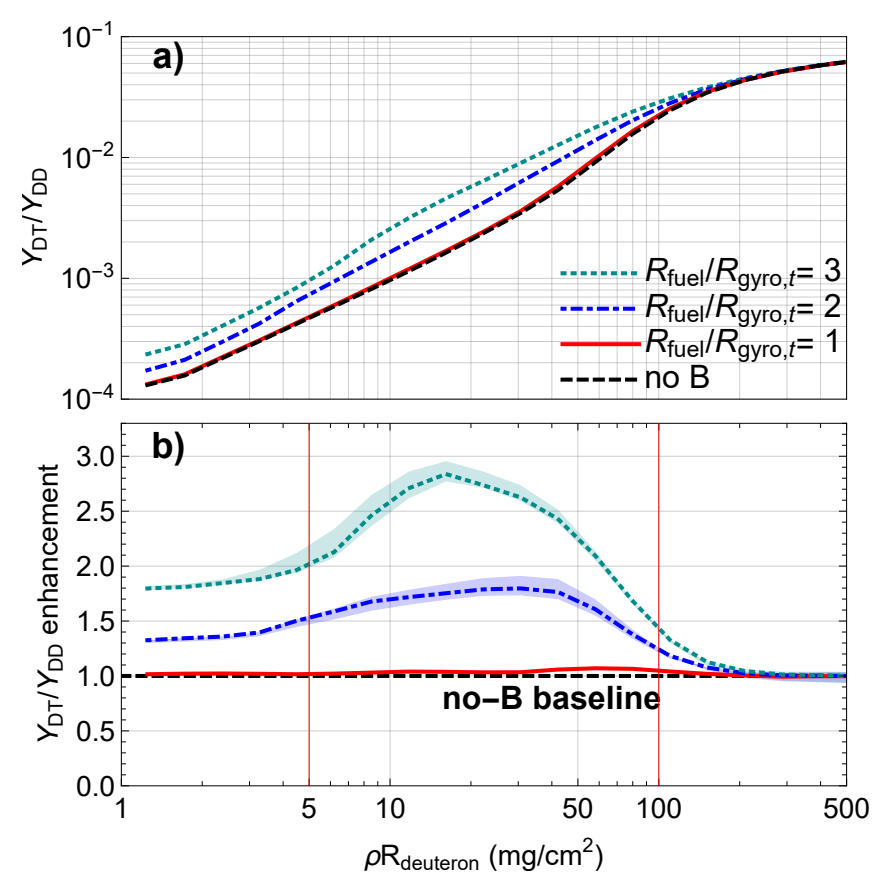

FIG. 2: a) Simulated secondary yield ratio $Y_{D T} / Y_{D D}$ as a function of deuteron $\rho \mathrm{R}$ and applied B-field. $R_{\text {fuel }} / R_{\text {gyro, },}$ of 0 (dashed, black), 1 (solid,red), 2 (dot-dashed, blue), and 3 (dotted, teal) corresponds to an applied B-field of $0.0,0.72,1.43$, and $2.14 \mathrm{kT}$, respectively. b) shows the $Y_{D T} / Y_{D D}$ enhancement factor relative to the no-B-field case (the horizontal black line). For this plasma, $T_{e}=T_{i}=5 \mathrm{keV}$, and the shaded areas in b) represent uncertainty introduced by $T_{e} \pm 0.5 \mathrm{keV}$ (for the cases $R_{\text {fuel }} / R_{\text {gyro, },}=2$ and 3 ). the additional magnetic confinement does not notably increase secondary reaction probability. These regimes are qualitative, as the exact thresholds are functions of ion and electron temperatures.

Secondary yield ratio is a sensitive probe of neutronaveraged magnetization in low-to-moderate- $\rho$ R ICF plasmas, provided fuel $\rho \mathrm{R}$ and plasma temperatures are wellcharacterized. The shaded areas in Fig. $2 \mathrm{~b}$ represent the yield ratio uncertainty introduced by $\pm 0.5 \mathrm{keV}$ uncertainty in electron temperature, a reasonable uncertainty from measurements of the x-ray continuum ${ }^{15}$. Between $\sim 5-100 \mathrm{mg} / \mathrm{cm}^{2}$ fuel $\rho R$, a $25 \%$ measurement uncertainty in $\rho \mathrm{R}$ (using a combination of x-ray imaging and proton spectrometers ${ }^{16}$ ) would contribute (approximately linearly) $25 \%$ uncertainty to $Y_{D T} / Y_{D D}$. These values are compared to typical neutron time-of-fight detector measurement uncertainty of 5\%-10\% ${ }^{17}$. These uncertainties are presented only to illustrate relative sensitivities to different plasma parameters, and Sec.V discusses in more details the limitations of the static model used here.

\section{SECONDARY NEUTRON SPECTRA}

Secondary DT neutron spectra along different lines of sight provide an additional diagnostic signature of plasma magnetization in an ICF implosion. DD-t with a large velocity component perpendicular to the applied B-field are preferentially confined (Fig.1), and the anisotropy in the confined DD-t population leads to anisotropy in the secondary DT neutron spectra along different lines of sight.

Figure 3a shows secondary DT-n spectra along lines of sight parallel (pole) and perpendicular (equator) to the applied B-field, for a uniform plasma with $R_{\text {fuel }} / R_{\text {gyro }, t}=2(\mathrm{~B}=1.43 \mathrm{kT})$. The DT-n spectrum parallel to the applied B-field is centrally peaked, whereas the DT-n spectrum perpendicular to the applied B-field has a central dip. For comparison, the DT-n spectra for the unmagnetized case are shown in dashed lines. The unmagnetized spectra are nearly identical as expected, as there is no imposed asymmetry in the system.

$\rho \mathrm{R}$ asymmetry (due to non-spherical implosion shape) can also lead to anisotropy in the secondary DT neutron spectra because DD-t now encounter different fuel $\rho \mathrm{R}$ along different directions. For example, the secondary DT spectra simulated for an oblate, unmagnetized plasma (Fig. 3b) show similar features as the neutron spectra for a spherical, magnetized plasma (Fig. 3a). Spectral shapes along different lines of sight provide useful diagnostic information about plasma magnetization that nonetheless must be interpreted in the context of other diagnostic information about the implosion. In this example, $\rho \mathrm{R}$ diagnostics ${ }^{16}$ along different lines of sight should measure much higher $\rho \mathrm{R}$ and also significant $\rho \mathrm{R}$ anisotropy for the oblate plasma in Fig. 3b. Similarly, $\mathrm{x}$-ray imaging can be used to confirm hot-spot shape. 

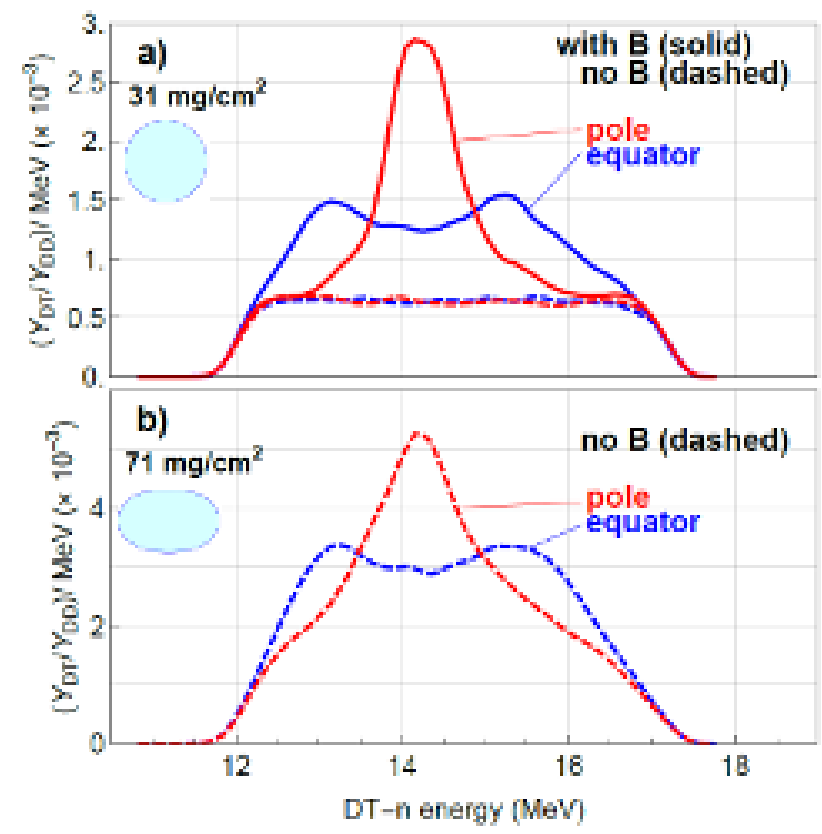

FIG. 3: Secondary DT neutron spectra along lines of sight parallel to the magnetic field (pole, red, pointing upward) and perpendicular to the magnetic field (equator, blue) for a uniform plasma with $T_{e}=5 \mathrm{keV}$. The solid lines are spectra simulated with an applied B-field of $1.43 \mathrm{kT}$, corresponding to $R_{\text {fuel }} / R_{\text {gyro }, t}=2$. The dashed lines are spectra simulated with no B-field. a) is the case for a spherical plasma with fuel $\rho R=31 \mathrm{mg} / \mathrm{cm}^{2}$, and $\mathrm{b}$ ) is the case for an oblate plasma with average fuel $\rho R=71 \mathrm{mg} / \mathrm{cm}^{2}$.

\section{v. DISCUSSION}

Sec.III identified two prerequisites to use secondary DT reactions as a magnetization diagnostic: 1) $R_{\text {fuel }} / R_{\text {gyro }, t} \geq 2$, and 2) fuel $\rho R<100 \mathrm{mg} / \mathrm{cm}^{2}$. Fig.4 shows the $Y_{D T} / Y_{D D}$ expected enhancement from an applied B-field for several representative ICF implosion types with different $R_{f u e l} / R_{\text {gyro,t }}$ and fuel $\rho R$. Capsule size, convergence, and temperature are based on previous implosions on NIF and OMEGA, when available. Electrons are magnetized in all cases (electron Hall parameter $>1$ ), and in higher-temperature plasmas thermal deuterons are also magnetized (ion Hall parameter $>1$ ).

Figure 4 shows that plasma magnetization can be best measured in large-diameter, moderate-convergence implosions. Smaller implosions can reach the same $R_{\text {fuel }} / R_{\text {gyro, } t}$ if they have a higher convergence ratio, but higher convergence ratio also leads to higher fuel $\rho R$. For example, a relatively-high- $\rho R$ indirect-drive warm implosion on the NIF (see Table I, "2mm ID compressive") can reach sufficient high $R_{\text {fuel }} / R_{\text {gyro,t }}$ to magnetically confine DD-t, but this will not lead to a useful magnetization signature because the fuel $\rho R$ is high enough that the DD-t are already collisionally confined.

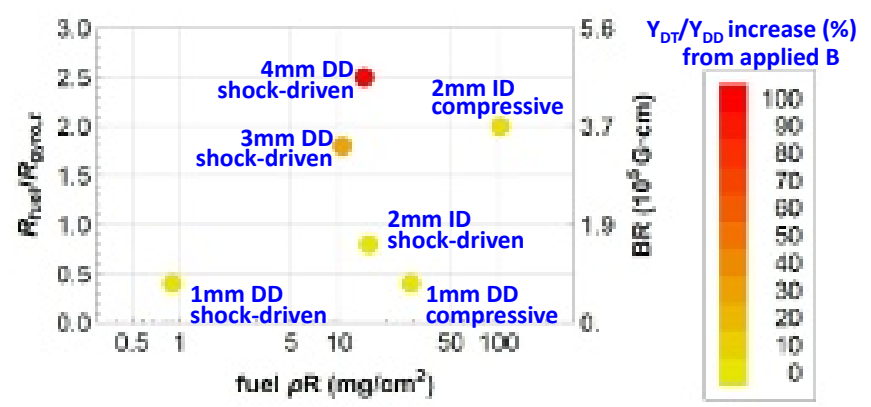

FIG. 4: $Y_{D T} / Y_{D D}$ increase (\%) from an applied B-field for different ICF implosion types as a function of $R_{\text {fuel }} / R_{\text {gyro,t }}$ and fuel $\rho \mathrm{R}$. DD and ID refer to direct-drive and indirect-drive implosions, respectively.

An important question is how well does this static Monte-Carlo model describe DD-t magnetic confinement in realistic ICF plasma conditions. Figure 5 shows the $Y_{D T} / Y_{D D}$ ratio using the Monte-Carlo (MC) model as a function of initial B-field for a 4-mm-diameter, directdrive implosion on NIF (see Table I, "4mm DD"). The model shows no change to the $Y_{D T} / Y_{D D}$ ratio until the initial B-field is $15-20 \mathrm{~T}$, followed by a linear increase. This is compared with the $Y_{D T} / Y_{D D}$ ratio simulated using the 2-D radiation-magnetohydrodynamic code LASNEX ${ }^{20}$ with DD-t transport through the magnetized fuel. Both the static MC model and the 2-D MHD simulations predict no change in the $Y_{D T} / Y_{D D}$ ratio until $\sim 20 \mathrm{~T}$, although the $Y_{D T} / Y_{D D}$ enhancement in the MHD simulation at $30 \mathrm{~T}$ is only half of that predicted by the static MC model. One reason may be that in the MHD implosion simulation, DD-t are generated during both shock rebound and compression, sampling a wide array of plasma conditions as compared to the static profiles assumed in the MC calculations. As compared to the B-field value at peak compression, the B-field value averaged over the fusion duration is reduced by $\sim 50 \%$.

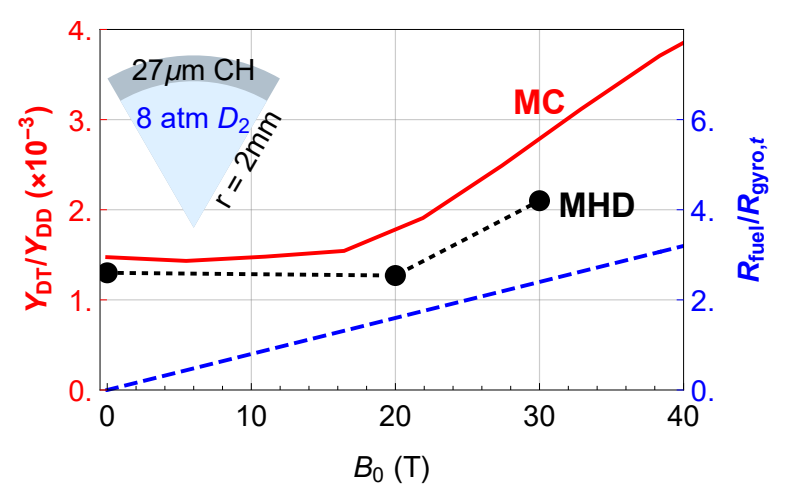

FIG. 5: $Y_{D T} / Y_{D D}$ as calculated from a Monte-Carlo (MC, solid,red) model using neutron-averaged quantities, and as simulated using LASNEX (MHD, dotted, black) for different initial B-field. The blue-dashed line indicates peak $R_{f u e l} / R_{\text {gyro,t }}$. 
TABLE I: ICF implosion parameters used in Fig.4. DD and ID refer to direct-drive and indirect-drive implosions, respectively. The column abbreviations are defined here: OD (outer capsule diameter), $T_{i}$ (ion temperature), $T_{e}$ (electron temperature), $\mathrm{CR}$ (convergence ratio), $\mathrm{B}_{0}$ (initial B-field), $\mathrm{B}_{f}$ (final compressed B-field), $C_{H, i}$ (thermal deuteron Hall parameter), and $C_{H, e}$ (electron Hall parameter).

\begin{tabular}{|c|c|c|c|c|c|c|c|c|c|c|c|}
\hline Implosion type & $\begin{array}{l}\mathrm{OD} \\
\mu \mathrm{m}\end{array}$ & $\begin{array}{l}\mathbf{T}_{\boldsymbol{i}} \\
\mathrm{keV}\end{array}$ & $\begin{array}{l}\mathbf{T}_{\boldsymbol{e}} \\
\mathrm{keV}\end{array}$ & CR & $\begin{array}{c}\boldsymbol{\rho} \mathbf{R} \\
\mathrm{mg} / \mathrm{cm}^{2}\end{array}$ & $\begin{array}{l}\mathbf{B}_{\mathbf{0}} \\
\mathrm{T}\end{array}$ & $\begin{array}{l}\mathbf{B}_{\boldsymbol{f}} \\
\mathrm{kT}\end{array}$ & $C_{H, i}$ & $C_{H, e}$ & $\boldsymbol{R}_{\text {fuel }} / \boldsymbol{R}_{\text {gyro,t }}$ & $\begin{array}{c}\text { BR } \\
10^{5} \mathrm{G} \cdot \mathrm{cm}\end{array}$ \\
\hline 4mm DD (shock driven) & 4000 & 8.9 & 4.0 & 7 & 14 & 30 & 1.6 & 1.4 & 42 & 2.5 & 4.4 \\
\hline 3mm DD (shock driven) & 3000 & 11.1 & 4.0 & 7 & 11 & 30 & 1.6 & 1.9 & 42 & 1.8 & 3.3 \\
\hline $2 \mathrm{~mm}$ ID (compressive) & 1816 & 2.5 & 2.5 & 13 & 103 & 30 & 5.1 & 0.04 & 5 & 2.0 & 3.6 \\
\hline $2 \mathrm{~mm}$ ID (shock driven) ${ }^{18}$ & 1870 & 3.5 & 3.5 & 5 & 15 & 30 & 0.8 & 0.1 & 11 & 0.8 & 1.4 \\
\hline $1 \mathrm{~mm}$ DD (compressive) ${ }^{5}$ & 860 & 3.2 & 3.0 & 20 & 28 & 8 & 3.2 & 0.03 & 4 & 0.4 & 0.7 \\
\hline $1 \mathrm{~mm}$ DD (shock driven) ${ }^{19}$ & 860 & 14.0 & 4.0 & 4 & 1 & 45 & 0.7 & 6.5 & 93 & 0.4 & 0.8 \\
\hline
\end{tabular}

\section{CONCLUSION}

The application of secondary DT reactions in diagnosing plasma magnetization in ICF implosions have been examined using Monte-Carlo models and 2-D MHD simulations. The dual prerequisites of sufficient B-field compression while remaining below the fuel $\rho R$ threshold limit the application of this technique to large-diameter, moderate-converge ICF implosions with the current Bfield generation platforms. However, when applicable, the combined diagnostic signatures of secondary yield ratio and neutron spectra are sensitive probes of neutronaveraged plasma magnetization.

\section{ACKNOWLEDGMENT}

The author thanks P. F. Knapp and P. F. Schmit (Sandia National Laboratories) for helpful discussions. This work was performed under the auspices of the U.S. Department of Energy by Lawrence Livermore National Laboratory under Contract DE-AC52-07NA27344.

\section{DATA AVAILABILITY STATEMENT}

The data that support the findings of this study are available from the corresponding author upon reasonable request.

\section{REFERENCES}

${ }^{1}$ J. Lindl, Physics of Plasmas 2, 3933 (1995).

${ }^{2}$ I. R. Lindemuth, Physics of Plasmas 22, 122712 (2015).

${ }^{3}$ L. J. Perkins, D. D.-M. Ho, B. G. Logan, G. B. Zimmerman, M. A. Rhodes, D. J. Strozzi, D. T. Blackfield, and S. A. Hawkins, Physics of Plasmas 24, 062708 (2017).

${ }^{4}$ J. D. Moody, A. Johnson, J. Javedani, E. Carroll, J. Fry, B. Kozioziemski, S. O. Kucheyev, B. G. Logan, B. B. Pollock, H. Sio, D. Strozzi, W. A. Stygar, V. Tang, and S. Winters, Physics of Plasmas 27, 112711 (2020).
${ }^{5}$ P. Y. Chang, G. Fiksel, M. Hohenberger, J. P. Knauer, R. Betti, F. J. Marshall, D. D. Meyerhofer, F. H. Séguin, and R. D. Petrasso, Phys. Rev. Lett. 107, 035006 (2011).

${ }^{6}$ M. Hohenberger, P.-Y. Chang, G. Fiksel, J. P. Knauer, R. Betti, F. J. Marshall, D. D. Meyerhofer, F. H. Séguin, and R. D. Petrasso, Physics of Plasmas 19, 056306 (2012).

${ }^{7}$ J. P. Knauer, O. V. Gotchev, P. Y. Chang, D. D. Meyerhofer, O. Polomarov, R. Betti, J. A. Frenje, C. K. Li, M. J.-E. Manuel, R. D. Petrasso, J. R. Rygg, and F. H. Séguin, Physics of Plasmas 17, 056318 (2010).

${ }^{8}$ M. D. Cable and S. P. Hatchett, Journal of Applied Physics 62 2233 (1987).

${ }^{9}$ P. F. Knapp, P. F. Schmit, S. B. Hansen, M. R. Gomez, K. D Hahn, D. B. Sinars, K. J. Peterson, S. A. Slutz, A. B. Sefkow, T. J. Awe, E. Harding, C. A. Jennings, M. P. Desjarlais, G. A. Chandler, G. W. Cooper, M. E. Cuneo, M. Geissel, A. J. HarveyThompson, J. L. Porter, G. A. Rochau, D. C. Rovang, C. L. Ruiz, M. E. Savage, I. C. Smith, W. A. Stygar, and M. C. Herrmann, Physics of Plasmas 22, 056312 (2015).

${ }^{10}$ B. Appelbe, J. Pecover, and J. Chittenden, High Energy Density Physics 22, 27 (2017)

${ }^{11} \mathrm{~S}$. Atzeni and J. Meyer-Ter-Vehn, The Physics of Inertial Fusion: Beam Plasma Interaction, Hydrodynamics, Hot Dense Matter, International Series of Monographs on Physics (Oxford University Press, 2004).

${ }^{12}$ C.-K. Li and R. D. Petrasso, Phys. Rev. Lett. 70, 3059 (1993).

${ }^{13}$ B. Appelbe and J. Chittenden, Physics of Plasmas 19, 073115 (2012).

${ }^{14}$ H. Brysk, Plasma Physics 15, 611 (1973).

${ }^{15}$ M. MacDonald, B. Kozioziemski, A. MacPhee, M. Schneider, J. Ayers, and D. Thorn, Journal of Instrumentation 14, P12009 (2019).

${ }^{16}$ F. H. Séguin, N. Sinenian, M. Rosenberg, A. Zylstra, M. J.-E. Manuel, H. Sio, C. Waugh, H. G. Rinderknecht, M. G. Johnson, J. Frenje, C. K. Li, R. Petrasso, T. C. Sangster, and S. Roberts, Review of Scientific Instruments 83, 10 D908 (2012).

${ }^{17}$ V. Y. Glebov, T. C. Sangster, C. Stoeckl, J. P. Knauer, W. Theobald, K. L. Marshall, M. J. Shoup, T. Buczek, M. Cruz, T. Duffy, M. Romanofsky, M. Fox, A. Pruyne, M. J. Moran, R. A. Lerche, J. McNaney, J. D. Kilkenny, M. J. Eckart, D. Schneider, D. Munro, W. Stoeffl, R. Zacharias, J. J. Haslam, T. Clancy, M. Yeoman, D. Warwas, C. J. Horsfield, J.-L. Bourgade, O. Landoas, L. Disdier, G. A. Chandler, and R. J. Leeper, Review of Scientific Instruments 81, 10D325 (2010), https://doi.org/10.1063/1.3492351.

${ }^{18}$ S. Le Pape, L. Divol, L. Berzak Hopkins, A. Mackinnon, N. B. Meezan, D. Casey, J. Frenje, H. Herrmann, J. McNaney, T. Ma, K. Widmann, A. Pak, G. Grimm, J. Knauer, R. Petrasso, A. Zylstra, H. Rinderknecht, M. Rosenberg, M. Gatu-Johnson, and J. D. Kilkenny, Phys. Rev. Lett. 112, 225002 (2014).

${ }^{19}$ A. Bose, "Magnetized shock-driven implosion platform at omega for studies of strong electron and ion magnetization," (2020), 62nd Annual Meeting of the APS Division of Plasma Physics.

${ }^{20}$ G. B. Zimmerman and W. L. Kruer, Comments Plasma Phys Controlled Fusion 2, 51 (1975). 


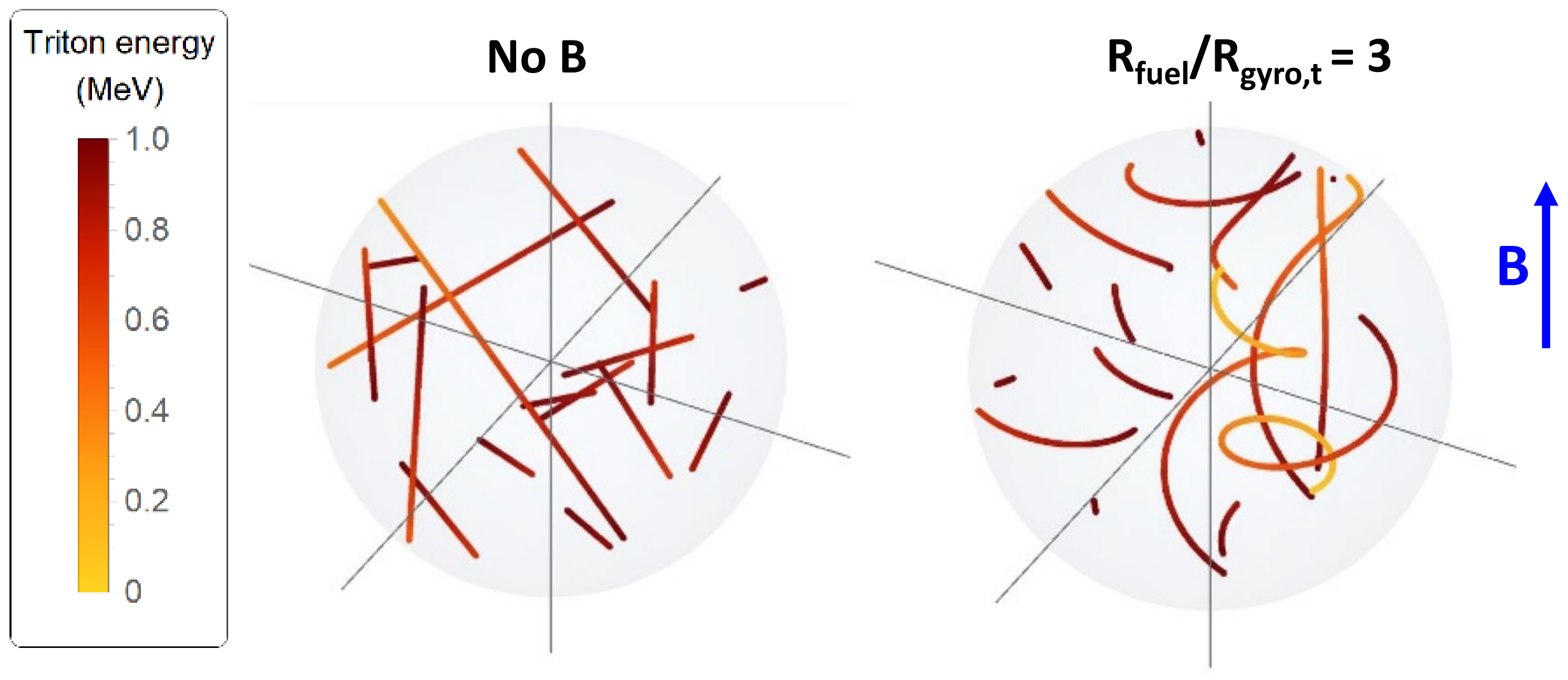



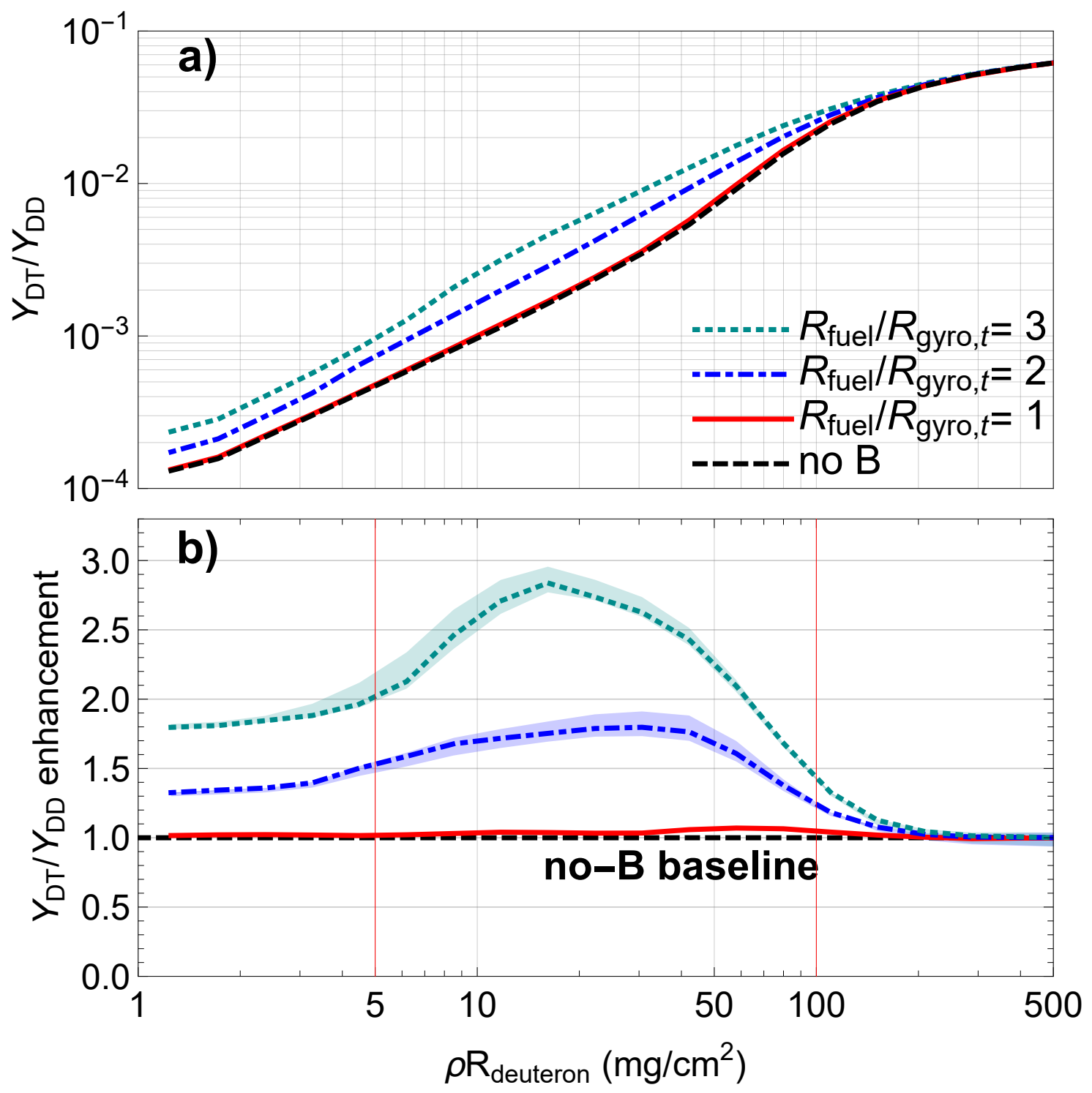


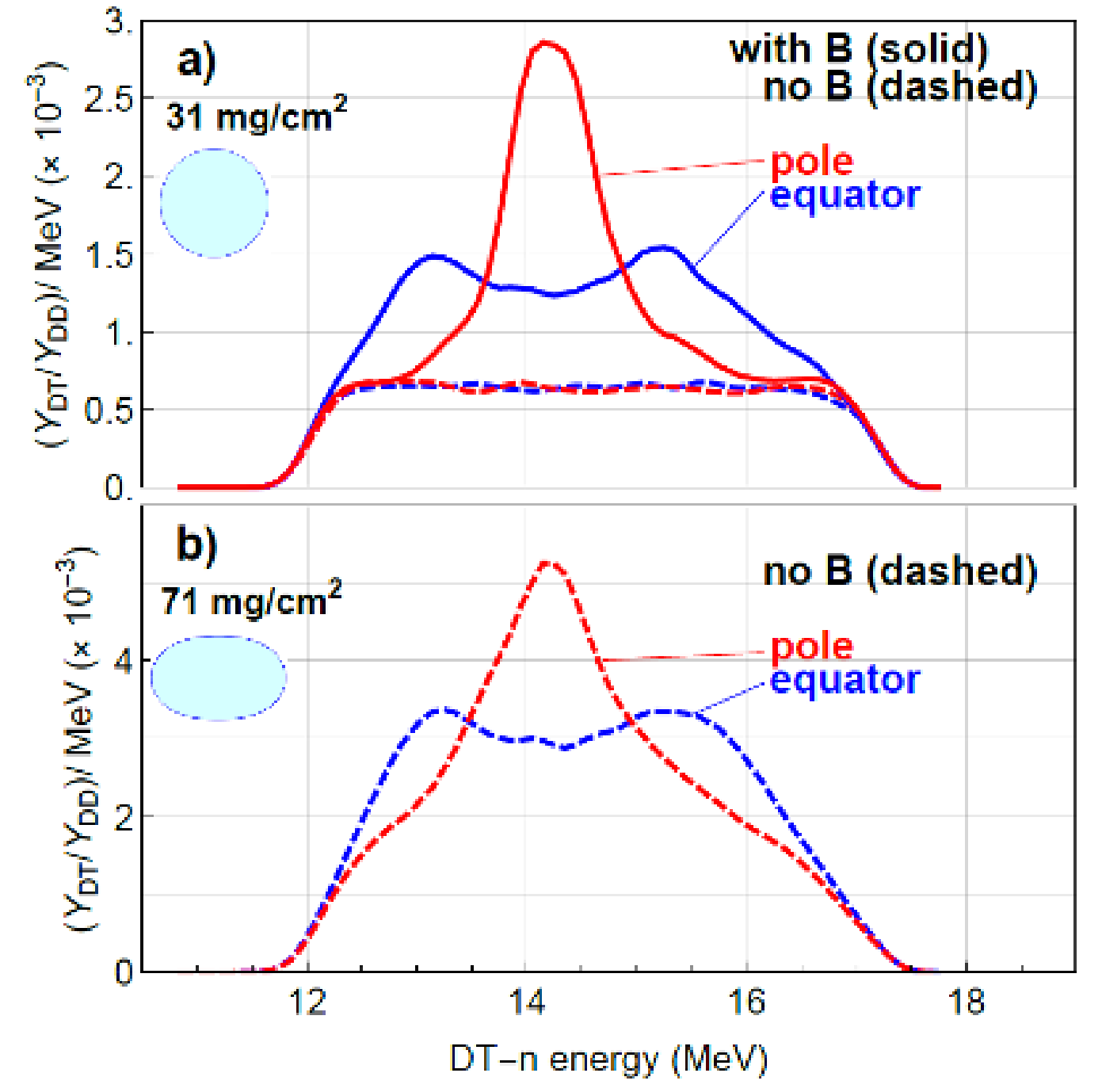




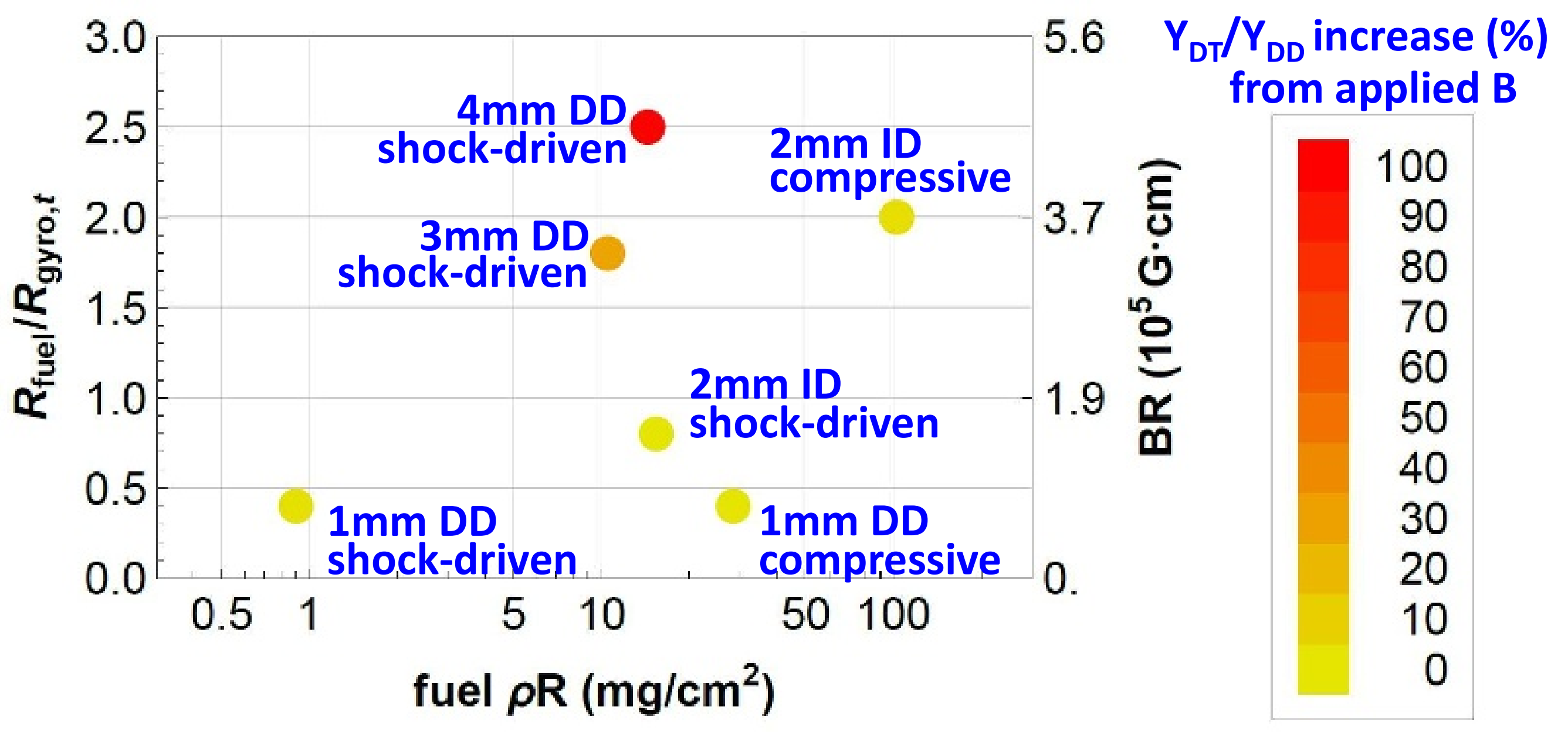




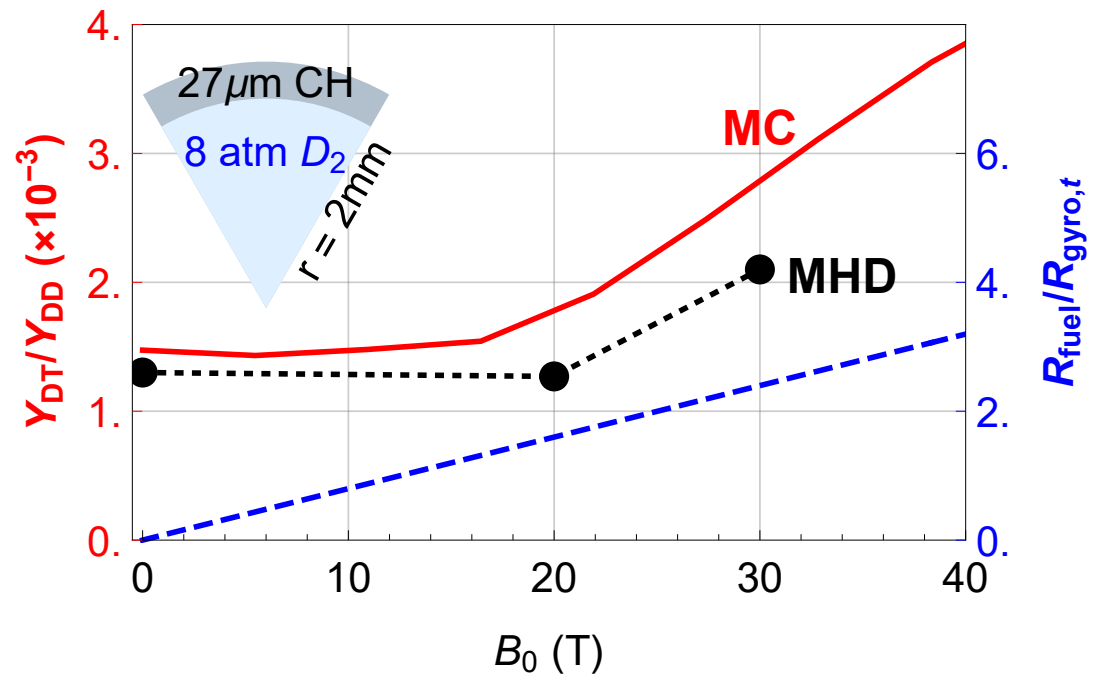

